Journal of Bangladesh Chemical Society, Vol. 25(2), 186-193, 2012

\title{
SYNTHESIS OF 2-BENZOYL-3,4,5,6-TETRASUBSTITUTEDBENZOIC ACID FROM TETRASUBSTITUTED PHTHALIC ANHYDRIDE USING ORGANOMETALICS
} PIZUSH KANTI BISWAS ${ }^{1 *}$, ENAMUL HUQ ${ }^{1}$, M. T. RAHMAN ${ }^{2}$ AND HUSNA PARVIN NUR ${ }^{3}$

${ }^{1 *}$ CRD, BCSIR Labs, Dhaka, ${ }^{1}$ C-11, Baily Ritz, 1, New Baily Road Dhaka, ${ }^{2}$ Ex. Professor Department of Chemistry, University of Dhaka, now at Brighton Sussex UK, ${ }^{3}$ BCSIR Dhaka 1205.

\section{Abstract}

The reactions of phenylcoppermagnesium and phenylmagnesium bromide reagents with perchlorophthalic anhydride were investigated. Along with 2-benzoyl-3,4,5,6tetrachlorobenzoic acid, five other substituted products were isolated and their structure were determined by detailed spectroscopic studies.

\section{Introduction}

Organometallic compounds are versatile reagents in organic synthesis ${ }^{2,3,4,5,6}$. The employment of organometallic compounds in the synthesis of keto acids have not been so extensively studied. Organocopper reagents are also versatile in organic synthesis s, $^{7,9,10,11}$. Rahman et $\mathrm{al}^{1}$. reported that the reaction of organocopper reagents such as organocoppermagnesium reagents $[2 \mathrm{RMgX}+\mathrm{CuI}]$ with cyclic anhydride give a mixture of keto acid and lactone ${ }^{1,12,13}$. The actual yield (83-98\%) of each product is dependent on the reaction conditions and on the type of organic groups employed in the copper reagent.<smiles>[R]c1c([R])c([R])c2c(c1[R])C(=O)O[C@H]2[Y19]([H])([H])I</smiles>

$$
\begin{aligned}
2,3,4: \text { a: } R_{1}=R_{2}=R_{3}=R_{4}=H \\
\text { b: } R_{1}=R_{2}=R_{3}=R_{4}=H
\end{aligned}
$$<smiles>[R]C(=O)c1c([R])c([R4])c([R3])c([R4])c1C(=O)O</smiles>

3<smiles>[R]C=C=C([R])c1c([R])c([R4])c2c(c1[R])C([R])(O)OC2=O</smiles>

1a: $\mathrm{R}_{5}=\mathrm{Ar}$

$1 \mathrm{~b}: \mathrm{R}_{5}=\mathrm{Me}$

The unsubstituted phthalic anhydride with the methylcoppermagnesium reagent produces only the 3,3-dimethylphthalide, whereas phenylcoppermagnesium reagent with tetraphenylphthalic anhydride, obtained $93 \%$ of the 2-benzoyltetraphenylbenzoic acid but all in its tautomaric lactol form. The aim of the present investigation was to examine the

* Author for correspondence; e-mail: pizushbiswas@gmail.com 
generality of the above hypothesis which states that the presence of electron-withdrawing substituents in the benzene ring of phthalic anhydride diverts the reaction of the latter with organocoppermagnesium reagents to the formation of 2-acylbenzoic acids in their lactol forms, regardless of the nature of the organic groups of the copper reagents used for this purpose. The anhydride chosen was tetrachlorophthalic anhydride and the copper reagent used was the phenylcoppermagnesium reagent. This reaction was compared with the reaction of phenylmagnesium halide and tetrachlorophthalic anhydride in order to gain further insight into the mechanism.

\section{Experimental}

Melting points were recorded on a Gallencamp melting point apparatus and are uncorrected. Silica gel 60 (E. Merck, 230-400 mesh ASTM) was used for column chromatography and silica gel 60 (E. Merck) ${ }_{\text {HF } 254+366}$ for tlc. Petrol refers to the AR petroleum ether (b.p $60-80^{\circ} \mathrm{C}$ ). IR spectra were recorded as "nujol mull" between sodium chloride plates in PYE UNICOM SP1025 IR-spectrophotometer. UV spectra were recorded in spectroscopic grade ethanol (Merck) with a Hitachi U-3501 instrument. Elemental analysis $(\mathrm{C}, \mathrm{H}, \mathrm{O})$ were conducted using the Perkin-Elmer 2400 series II elemental analyzer, $500 \mathrm{MHz}{ }^{1} \mathrm{H}$ NMR and $125.5 \mathrm{MHz}{ }^{13} \mathrm{C}$ NMR were recorded in $\mathrm{CDCl}_{3}$ and DMSO- $\mathrm{d}_{6}$ solution with a VARIAN UNIT XL-500 NMR spectrometer (chemical shift $\delta$ in ppm and $\mathrm{J}$ in $\mathrm{Hz}$ ). Mass spectra were recorded with the help of GCMS Finnegan 1020 automated GC/MS system. Analytical samples were routinely dried over blue silica gel in vacuum at room temperature.

The phenylmagnesium Bromide reagent ${ }^{14}$ was prepared from Grignard grade Mg-turning and bromobenzene. The phenylmagnesium bromide solution prepared in ether was brownish in colour and gives a positive Gilman colour Test- $\mathrm{I}^{15}$. The phenylcoppermagnesium reagent ${ }^{16}$ was prepared by the addition of copper (1) iodide to a cooled solution $\left(-5\right.$ to $\left.-15^{\circ} \mathrm{C}\right)$ of phenylmagnesium reagent in an oxygen free dry nitrogen atmosphere. The preparation of phenylcoppermagnesium reagent was confirmed by Gilman colour Test- ${ }^{15}$. No blue/green colour developed indicating that all of the phenylmagnesium bromide had reacted.

\section{Results and Discussion}

The reaction of the phenylcoppermagnesium reagent $1 \mathbf{a}(2.5 \mathrm{eq}$.) with tetrachlorophthalic anhydride 5(1eq.) gives a mixture of compounds 6-12 (Scheme-2). 


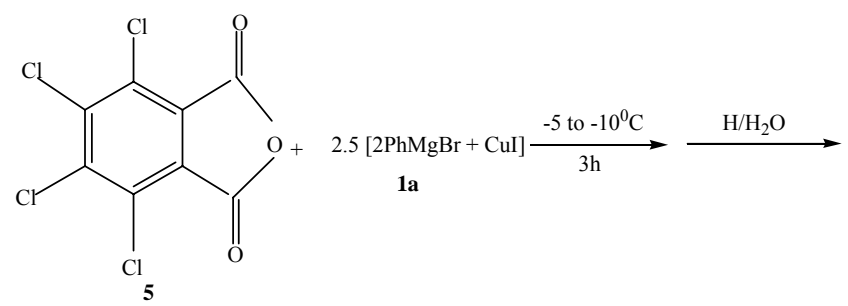<smiles>O=C(O)c1c(Cl)c(Cl)c(Cl)c(Cl)c1C(=O)c1c(Cl)c(Cl)c(Cl)c(C(=O)[PH2+]c2ccccc2)c1-c1ccccc1</smiles><smiles>O=C(c1c(Cl)c(Cl)c(C(=O)[PH2+])c(C(=O)c2c(Cl)c(Cl)c(Cl)c(Cl)c2Cl)c1Cl)c1c([PH2+])c(Cl)c(Cl)c(Cl)c1C(=O)c1ccccc1</smiles><smiles>O=C1OC(c2ccccc2)(c2ccccc2)c2c(Cl)c(Cl)c(Cl)c(Cl)c21</smiles>

The results showed that the reaction is more complex than corresponding reactions with the unsubstituted or perarylated phthalic ${ }^{1}$ anhydrides and give a complex mixture of products. Secondly 2-aroyltetrachlorobenzoic acid is not obtained in its ring tautomeric lactol form as expected but instead as its open-chain tautomer in sharp contrast with the corresponding reaction with perphenilatedphthalic anhydride ${ }^{12}$. Thirdly in addition to the usual nucleophilic addition to one of the carbonyl carbons of tetrachlorophthalic anhydride, some metal-halogen exchange reactions have taken place presumably because of the activating effects produced by the perchlorination of the ring. And finally, some products are formed by the decarboxylation of the highly chlorenated benzoic acid. 
It was interesting to compare these results with those obtained from the corresponding reactions with phenylmagnesium bromide and tetrachlorophthalic anhydride. The results indicated that a similar mixture of products are also obtained with the exception that the yields of 2-benzoyl-3,4,5,6-tetrachlorobenzoic acid (6) in the reaction involving the cuprate reagent is about 10-times higher than in the reaction with the Grignard reagent. Doubtless in the latter reaction, Grignard reagent further reacted with (6) to form the corresponding phthalide (12). This phthalied was not obtained in the cuprate reaction indicating that a cuprate reagent of the type $[2 \mathrm{PhMgBr}+\mathrm{CuI}]$ does not reacted with the carbonyl group of (6).

Tale 1. Comparison of the yield (\%) of the products using [2PhMgBr $+\mathrm{CuI}]$ and $\mathrm{PhMgBr}$ reagents with perchlorophthalic anhydride

\begin{tabular}{|l|l|l|}
\hline Products & $\begin{array}{l}\text { Yield (\%) } \\
{[2 \mathrm{PhMgBr}+\mathrm{CuI}]}\end{array}$ & $\begin{array}{l}\text { Yield (\%) } \\
\text { PhMgBr }\end{array}$ \\
\hline 6 & 70 & 7 \\
\hline 7 & 5 & 28 \\
\hline 8 & 5 & 29 \\
\hline 9 & 5 & 14 \\
\hline 10 & 3.5 & 5 \\
\hline 11 & 3.5 & 2 \\
\hline 12 & - & 10 \\
\hline
\end{tabular}

\section{Conclusion}

This investigation has provided a new method for the preparation of difficultly obtainable, 2-benzoyl-3,4,5,6-tetrachlorobenzoic acid (6). Because many halogenated, especially chlorinated aromatic compounds are biologically active; compounds obtained in this study offer interesting biological possibilities.

\section{Reaction procedure}

Tetrachlorophthalic anhydride $(0.01$ mole $)$ was added all in one portion to phenylcoppermagnesium reagent / phenylmagnesium bromide reagent prepared from 0.05 mole of bromobenzene $0.05 \mathrm{~g}$-at of $\mathrm{Mg}$ and 0.025 mole of $\mathrm{CuI}$ (in the case of phenylcoppermagnesium reagent) in $10 \mathrm{ml}$ of ether with constant stirring under dry nitrogen atmosphere. The mixture was stirred for 2.5 hours at $-5^{0} \mathrm{C}$ and then allowed to stand at room temperature and hydrolyzed with $1 \mathrm{~N} \mathrm{HCl}(100 \mathrm{ml})$. A grayish-white solid precipitateted which was removed by filtering through glass-wool. The filtrate was extracted thrice $(3 \times 50 \mathrm{ml})$ with diethyl ether. The combined ether extract was further extracted with 5\% aqueous sodium bicarbonate and the alkali extract was acidified with $38 \% \mathrm{HCl}$. A milky-white colloidal suspension was obtained which was cooled in refrigerator overnight to obtain white crystals. The crystals of were dried under vacuum for several hours. The ether extract (neutral part) that remains after alkali extraction was dried under vacuum to give a yellowish gummy mass. The neutral part was subjected to 
column chromatography over silica gel (E. Merck 230-400 mesh). Petroleum ether (60$80^{\circ} \mathrm{C}$ ) and benzene were used as solvents for making the column. The adsorbent was supported on a plug of Glass-wool placed at the bottom of the column. Exclusion of air bubble was affected by making the column as quickly as possible. The surface of the column was covered with glass-wool also. The mixture of the products in concentrated form was applied at the top of the column. The mixture was allowed to be adsorbed on the surface of the column and eluted with the solvent. The fractions which came out of the column, were collected in small portion $(20 \mathrm{ml})$ in conical flasks, concentrated and were followed by tlc to check the progress of separations. The column was gradually eluted with solvents of increasing polarity by using benzene and petroleum ether (60$80^{\circ} \mathrm{C}$ ) solvent mixture of (1:9), (1:4), (3:7) ratios.

2-Benzoyl-3,4,5,6-tetrachlorobenzoic acid (6): White crystals m.p.: $193-95^{0} \mathrm{C}$; yield: $1.25 \mathrm{~g}(70 \%), \mathrm{R}_{\mathrm{f}} 0.50$ (silicagel, benzene : pet. ether 2:3). IR: $v=3520$ (s, CO-H stretching), 3000 (s, Ar-H stretching), $1760(\mathrm{~s},>\mathrm{C}=\mathrm{O}), 1600$ and $1580(\mathrm{w},>\mathrm{C}=\mathrm{C}<$, aromatic ring), $1230(\mathrm{w}, \mathrm{C}-\mathrm{O}), 800$ and $790\left(\mathrm{w}, \mathrm{Ar}-\mathrm{H}\right.$, bending) $\mathrm{cm}^{-1} ; \mathrm{UV}: \lambda_{\max }(\log \varepsilon)=$ 253 (4.37), 165 (3.45) nm; ${ }^{1} \mathrm{H}$ NMR (DMSO-d $\left.{ }_{6}, \delta, 500 \mathrm{MHz}\right) ; 9.30(1 \mathrm{H}, \mathrm{s}$, carboxylic proton), $7.72\left(2 \mathrm{H}, \mathrm{t}, \mathrm{J}=7.6, \mathrm{H}-2^{\prime}, 6^{\prime}\right), 7.51\left(2 \mathrm{H}, \mathrm{t}, \mathrm{J}=7.7, \mathrm{H}-3^{\prime}, 5^{\prime}\right), 7.62(1 \mathrm{H}, \mathrm{d}, \mathrm{J}=7.8$, H-4') ppm; ${ }^{13} \mathrm{C}$ NMR (DMSO-d $\left.6, \delta, 125.5 \mathrm{MHz}\right): 192.15(>\mathrm{C}=\mathrm{O}), 185.80(\mathrm{COOH}), 139.25(\mathrm{C}-$ 1), 138.25 (C-2), 135.15 (C-3), 134.85 (C-4), 134.12 (C-5), 134.39 (C-6), 129.17 (C-1'), $128.75\left(\mathrm{C}-2^{\prime}, 6^{\prime}\right), 128.25\left(\mathrm{C}-3^{\prime}, 5^{\prime}\right), 127.76\left(\mathrm{C}-4^{\prime}\right) \mathrm{ppm}$; MS (EI): $\mathrm{m} / \mathrm{e}=362\left(52 \%, \mathrm{M}^{+}\right), 317$ (23\%, M $-\mathrm{COOH}), 285\left(82 \%, \mathrm{M}^{+}-\mathrm{Ph}\right), 222\left(3 \%, \mathrm{M}^{+}-\mathrm{Ph}-\mathrm{CO}-\mathrm{Cl}\right), 177\left(42 \%, \mathrm{M}^{+}-\mathrm{COPh}-\right.$ $\mathrm{COOH}-\mathrm{Cl}), 149$ (23\%, M $-\mathrm{COOH}-\mathrm{CO}-4 \mathrm{Cl}), 142$ (76\%, M $-\mathrm{COPh}-\mathrm{COOH}-2 \mathrm{Cl}), 105$ $\left(100 \%,{ }^{+} \mathrm{COPh}\right), 77\left(100 \%, \mathrm{Ph}^{+}\right)$. Isotopic pattern, $\mathrm{m} / \mathrm{e}=370\left(17 \%, \mathrm{M}^{+}\right), 325\left(8 \%, \mathrm{M}^{+}\right.$$\mathrm{COOH}), 293\left(27 \%, \mathrm{M}^{+}-\mathrm{Ph}\right)$.

Anal. Calcd. for $\mathrm{C}_{14} \mathrm{H}_{6} \mathrm{O}_{3} \mathrm{Cl}_{4}$ : C, 46.20; H, 1.66; O, 13.19. Found: C, 46.12; H, 1.59; O, 13.11 .

2-Phenyl-3,4,5-trichlorobenzophenone (7): White crystals m.p.: $120-21^{0} \mathrm{C}$, yield: $0.33 \mathrm{~g}$ $(5 \%), \mathrm{R}_{\mathrm{f}} 0.62$ (silicagel, benzene : pet. ether 2:3). IR: $v=3000$ (s, Ar-H stretching), 1690 (s, $>\mathrm{C}=\mathrm{O}$ ), 1603 and $1582(\mathrm{w},>\mathrm{C}=\mathrm{C}<$, aromatic ring), 850 and 780 (w, Ar-H, bending) $\mathrm{cm}^{-1}$; UV: $\lambda_{\max }(\log \varepsilon)=250(4.35), 172(3.62) \mathrm{nm} ;{ }^{1} \mathrm{H} \mathrm{NMR}\left(\mathrm{CDCl}_{3}, \delta, 500 \mathrm{MHz}\right) ; 7.73$ $(1 \mathrm{H}, \mathrm{s}, \mathrm{H}-6), 7.20\left(2 \mathrm{H}, \mathrm{d}, \mathrm{J}=7.3, \mathrm{H}-2^{\prime}, 6^{\prime}\right), 6.92(2 \mathrm{H}, \mathrm{t}, \mathrm{J}=7.2, \mathrm{H}-3,5), 7.05(1 \mathrm{H}, \mathrm{d}, \mathrm{J}=7.4$, H-4), 7.58 (2H, d, J=7.1, H-2",6"), 7.35 (2H, t, J=7.0, H-3",5"), 7.49 (1H, d, J=7.3, H-4") ppm; ${ }^{13} \mathrm{C}$ NMR $\left(\mathrm{CDCl}_{3}, \delta, 125.5 \mathrm{MHz}\right): 193.18(>\mathrm{C}=\mathrm{O}), 141.48\left(\mathrm{C}-1^{\prime}\right), 138.27(\mathrm{C}-2)$, 136.12 (C-3), 135.81 (C-4), 134.43 (C-5), 128.52 (C-6), 133.90 (C-1'), 129.97 (C-2', 6'), $129.32\left(\mathrm{C}-3^{\prime}, 5^{\prime}\right), 129.27\left(\mathrm{C}-4^{\prime}\right), 128.58\left(\mathrm{C}-1^{\prime \prime}\right), 128.41\left(\mathrm{C}-2^{\prime \prime}, 6^{\prime \prime}\right), 128.38\left(\mathrm{C}-3^{\prime \prime}, 5^{\prime \prime}\right)$, $127.63\left(\mathrm{C}-4^{\prime \prime}\right)$ ppm: MS (EI): $\mathrm{m} / \mathrm{e}=360\left(19 \%, \mathrm{M}^{+}\right), 325\left(5 \%, \mathrm{M}^{+}-\mathrm{Cl}\right), 283\left(17 \%, \mathrm{M}^{+}-\mathrm{Ph}\right)$,

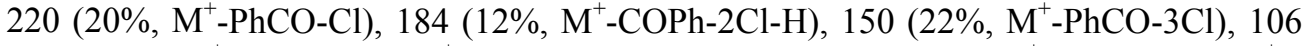
$\left(100 \%, \mathrm{PhCO}^{+}\right), 77\left(80 \%, \mathrm{Ph}^{+}\right)$. Isotopic pattern, $\mathrm{m} / \mathrm{e}=366\left(6 \%, \mathrm{M}^{+}\right), 331\left(2 \%, \mathrm{M}^{+}\right.$$\mathrm{Cl}), 289\left(6 \%, \mathrm{M}^{+}-\mathrm{Ph}\right)$.

Anal. Calcd. for $\mathrm{C}_{19} \mathrm{H}_{11} \mathrm{OCl}_{3}$ : C, 63.1; H, 3.07; O, 4.43 Found: C, 63.02; H, 3.01; O, 4.37. 
5-Phenyl-2,3,4-trichlorobenzophenone (8): Brownish crystals m.p.: $115-20^{\circ} \mathrm{C}$, yield $0.33 \mathrm{~g}(5 \%), \mathrm{R}_{\mathrm{f}} 0.60$ (silicagel, benzene : pet. ether 2:3). IR: $v=2985$ (s, Ar-H stretching), $1695(\mathrm{~s},>\mathrm{C}=\mathrm{O}), 1600$ and $1550(\mathrm{w},>\mathrm{C}=\mathrm{C}<$, aromatic ring), 855 and $750(\mathrm{w}, \mathrm{Ar}-\mathrm{H}$, bending) $\mathrm{cm}^{-1}$; UV: $\lambda_{\max }(\log \varepsilon)=252(3.82), 169(4.12) \mathrm{nm} ;{ }^{1} \mathrm{H}$ NMR $\left(\mathrm{CDCl}_{3}, \delta, 500\right.$ $\mathrm{MHz}) ; 7.64$ (1H, s, H-6), 7.25 (2H, d, J=7.3, H-2',6'), 7.15 (2H, t, J=7.3, H-3',5'), 6.80 $\left(1 \mathrm{H}, \mathrm{d}, \mathrm{J}=7.0, \mathrm{H}-4^{\prime}\right), 7.50\left(2 \mathrm{H}, \mathrm{d}, \mathrm{J}=7.2, \mathrm{H}-2^{\prime \prime}, 6^{\prime \prime}\right), 7.30\left(2 \mathrm{H}, \mathrm{t}, \mathrm{J}=7.2, \mathrm{H}-3^{\prime \prime}, 5^{\prime \prime}\right), 7.40(1 \mathrm{H}$, d, J=7.3, H-4") ppm; ${ }^{13} \mathrm{C}$ NMR $\left(\mathrm{CDCl}_{3}, \delta, 125.5 \mathrm{MHz}\right): 192.90(>\mathrm{C}=\mathrm{O}), 141.33(\mathrm{C}-1)$, 138.25 (C-2), 136.10 (C-3), 135.21 (C-4), 134.40 (C-5), 128.15 (C-6), 133.92 (C-1'), $129.30\left(\mathrm{C}-2^{\prime}, 6^{\prime}\right), 129.20\left(\mathrm{C}-3^{\prime}, 5^{\prime}\right), 128.50\left(\mathrm{C}-4^{\prime}\right), 128.45\left(\mathrm{C}-1^{\prime \prime}\right), 128.40\left(\mathrm{C}-2^{\prime \prime}, 6^{\prime \prime}\right), 128.30$ $\left(\mathrm{C}-3^{\prime \prime}, 5^{\prime \prime}\right), 127.60\left(\mathrm{C}-4^{\prime \prime}\right) \mathrm{ppm}$; MS (EI): m/e = $360\left(25 \%, \mathrm{M}^{+}\right), 325\left(10 \%, \mathrm{M}^{+}-\mathrm{Cl}\right), 283$ $\left(15 \%, \mathrm{M}^{+}-\mathrm{Ph}\right), 220\left(25 \%, \mathrm{M}^{+}-\mathrm{PhCO}-\mathrm{Cl}\right), 184\left(10 \%, \mathrm{M}^{+}-\mathrm{PhCO}-2 \mathrm{Cl}-\mathrm{H}\right), 150(20 \%, \mathrm{M}-$ $\left.\mathrm{PhCO}^{+}-3 \mathrm{Cl}\right), 106\left(100 \%, \mathrm{PhCO}^{+}\right), 77\left(81 \%, \mathrm{Ph}^{+}\right)$. Isotopic pattern, $\mathrm{m} / \mathrm{e}=366\left(8 \%, \mathrm{M}^{+}\right)$, $331\left(3 \%, \mathrm{M}^{+}-\mathrm{Cl}\right), 289\left(5 \%, \mathrm{M}^{+}-\mathrm{Ph}\right)$.

Anal. Calcd. for $\mathrm{C}_{19} \mathrm{H}_{11} \mathrm{OCl}_{3}$ : C, 63.11; H, 3.07; O, 4.43 Found: C, 63.05; H, 3.03; O, 4.32 1,2-Dibenzoyl-3,4,5,6-tetrachlorobenzene (9): Gummy mass, yield: $0.43 \mathrm{~g}(5 \%), \mathrm{R}_{\mathrm{f}} 0.55$ (silicagel, benzene : pet. ether 2:3). IR: $v=3000$ (m, Ar-H stretching), $1780(\mathrm{~s},>\mathrm{C}=\mathrm{O})$, 1600 and 1580 (s, $>\mathrm{C}=\mathrm{C}<$, aromatic ring), 820 and 800 (w, Ar- $\mathrm{H}$, bending) $\mathrm{cm}^{-1}$; UV: $\lambda_{\max }(\log \varepsilon)=251(4.32), 167(4.35) \mathrm{nm} ;{ }^{1} \mathrm{H} \mathrm{NMR}\left(\mathrm{CDCl}_{3}, \delta, 500 \mathrm{MHz}\right) ; 7.75(4 \mathrm{H}, \mathrm{d}$, $\mathrm{J}=7.4, \mathrm{C}, \mathrm{H}-2,6), 7.45$ (4H, t, J=7.4, C,H-3,5), $7.59(2 \mathrm{H}, \mathrm{t}, \mathrm{J}=7.2, \mathrm{C}, \mathrm{H}-4), \mathrm{ppm} ;{ }^{13} \mathrm{C}$ NMR $\left(\mathrm{CDCl}_{3}, \delta, 125.5 \mathrm{MHz}\right): 191.91(>\mathrm{C}=\mathrm{O}), 139.15(\mathrm{C}-1,6), 135.57(\mathrm{C}-2,5), 134.47(\mathrm{C}-3,4)$, 129.91 (C-1), 128.89 (C-2,6), 128.79 (C-3,5), 128.29 (C-4) ppm; MS (EI): m/e = 422 $\left(18 \%, \mathrm{M}^{+}\right), 347\left(5 \%, \mathrm{M}^{-} \mathrm{Cl}\right), 345\left(11 \%, \mathrm{M}^{+}-\mathrm{Ph}\right), 254\left(6 \%, \mathrm{M}^{+}-\mathrm{PhCO}-\mathrm{CO}-\mathrm{Cl}\right), 218(3 \%$, $\mathrm{M}^{+}$-PhCO-CO-2Cl), $105\left(80 \%, \mathrm{PhCO}^{+}\right), 77\left(100 \%, \mathrm{Ph}^{+}\right)$. Isotopic pattern, $\mathrm{m} / \mathrm{e}=430(6$ $\left.\%, \mathrm{M}^{+}\right), 393\left(2 \%, \mathrm{M}^{+}-\mathrm{Cl}\right), 353\left(4 \%, \mathrm{M}^{+}-\mathrm{Ph}\right)$.

Anal. Calcd. for $\mathrm{C}_{20} \mathrm{H}_{10} \mathrm{O}_{2} \mathrm{Cl}_{4}$ : C, 56.64; H, 2.38; O, 7.55 Found: C, 56.55; H, 2.28; O, 7.43

1,2-Dibenzoyl-3,4,5,6-tetrachlorobenzene (10): White crystals m.p.: $148-50^{\circ} \mathrm{C}$, yield $0.25 \mathrm{~g}(3.5 \%), \mathrm{R}_{\mathrm{f}} 0.42$ (silicagel, benzene : pet. ether 2:3). IR: $v=3000(\mathrm{~s}, \mathrm{Ar}-\mathrm{H}$ stretching), $1690(\mathrm{~s},>\mathrm{C}=\mathrm{O}$ ), 1600 and $1585(\mathrm{w},>\mathrm{C}=\mathrm{C}<$, aromatic ring), 810 and 798 (w, Ar-H, bending) $\mathrm{cm}^{-1}$; UV: $\lambda_{\max }(\log \varepsilon)=251(4.36), 162(3.97) \mathrm{nm} ;{ }^{1} \mathrm{H} \mathrm{NMR}\left(\mathrm{CDCl}_{3}, \delta\right.$, $500 \mathrm{MHz}$ ); 7.66 (1H, s, H-6), 7.72 (4H, d, J=7.4, H-2,6), 7.46 (4H, t, J=7.4, H-3,5), 7.60 $(2 \mathrm{H}, \mathrm{q}, \mathrm{H}-4), \mathrm{ppm} ;{ }^{13} \mathrm{C} \mathrm{NMR}\left(\mathrm{CDCl}_{3}, \delta, 125.5 \mathrm{MHz}\right): 193.07(>\mathrm{C}=\mathrm{O}), 139.25(\mathrm{C}-1)$, 141.9 (C-2), 137.27 (C-3), 136.35 (C-4), 135.72 (C-5), 129.20 (C-6), 129.33 (C-1), 128.77 (C-2,6), 128.70 (C-3.5), 128.64 (C-4) ppm; MS (EI): m/e = $388(8 \%, \mathrm{M})^{+}, 353$ $\left(3 \%, \mathrm{M}^{+}-\mathrm{Cl}\right), 311\left(18 \%, \mathrm{M}^{+}-\mathrm{Ph}\right), 283\left(2 \%, \mathrm{M}^{+}-\mathrm{PhCO}\right), 248\left(2 \%, \mathrm{M}^{+}-\mathrm{PhCO}-\mathrm{Cl}\right), 220(8 \%$, $\mathrm{M}^{+}$-PhCO-CO-Cl), 184 (4\%, M $\left.-\mathrm{PhCO}-\mathrm{CO}-2 \mathrm{Cl}\right), 150$ (11\%, M $\left.-\mathrm{PhCO}-\mathrm{CO}-3 \mathrm{Cl}\right), 105$ $\left(67 \%, \mathrm{PhCO}^{+}\right), 77\left(100 \%, \mathrm{Ph}^{+}\right)$. Isotopic pattern, $\mathrm{m} / \mathrm{e}=394\left(3 \%, \mathrm{M}^{+}\right), 357\left(1 \%, \mathrm{M}^{+}\right.$$\mathrm{Cl}), 317\left(6 \%, \mathrm{M}^{+}-\mathrm{Ph}\right)$. 
Anal. Calcd. for $\mathrm{C}_{20} \mathrm{H}_{11} \mathrm{O}_{2} \mathrm{Cl}_{3}: \mathrm{C}, 61.65 ; \mathrm{H}, 2.85 ; \mathrm{O}, 8.22$ Found: $\mathrm{C}, 61.58 ; \mathrm{H}, 2.79 ; \mathrm{O}$, 8.17

3',3'-Diphenyl-4,5,6-trichlorophthalide (11): White crystals m.p.: $135-40^{\circ} \mathrm{C}$, yield $0.25 \mathrm{~g}$ (3.5\%), $\mathrm{R}_{\mathrm{f}} 0.46$ (silicagel, benzene : pet. ether 2:3). IR: $v=2995$ (s, Ar-H stretching), $1685(\mathrm{~s},>\mathrm{C}=\mathrm{O}), 1610$ and $1580(\mathrm{w},>\mathrm{C}=\mathrm{C}<$, aromatic ring), 800 and $790(\mathrm{w}, \mathrm{Ar}-\mathrm{H}$, bending) $\mathrm{cm}^{-1}$; UV: $\lambda_{\max }(\log \varepsilon)=250(3.80), 173(3,42) \mathrm{nm} ;{ }^{1} \mathrm{H}$ NMR $\left(\mathrm{CDCl}_{3}, \delta, 500\right.$ $\mathrm{MHz}) ; 7.64$ (1H, s, H-7), 7.72 (4H, d, J=7.4, H-2,6), 7.46 (4H, t, J=7.2, H-3,5), $7.61(2 \mathrm{H}$, q, H-4), ppm; ${ }^{13} \mathrm{C}$ NMR $\left(\mathrm{CDCl}_{3}, \delta, 125.5 \mathrm{MHz}\right): 189.13$ (phthalide), 141.10 (C-1), 139.34 (C-2), 137.20 (C-4), 136.33 (C-5), 135.70 (C-6), 71.9 (C-3), 128.75 (C-1), 128.68 (C2,6), 128.62 (C-3,5), 128.55 (C-4) ppm; MS (EI): m/e = $388\left(10 \%, \mathrm{M}^{+}\right), 353\left(5 \%, \mathrm{M}^{+}-\right.$ $\mathrm{Cl}), 311\left(18 \%, \mathrm{M}^{+}-\mathrm{Ph}\right), 283\left(5 \%, \mathrm{M}^{+}-\mathrm{PhCO}\right), 248\left(5 \%, \mathrm{M}^{+}-\mathrm{PhCO}-\mathrm{Cl}\right), 220\left(10 \%, \mathrm{M}^{+}-\right.$ PhCO-CO-Cl), 184 (5\%, M+ $-\mathrm{PhCO}-\mathrm{CO}-2 \mathrm{Cl}), 150$ (10\%, M+PhCO-CO-3Cl), 105 (60\%, $\left.\mathrm{PhCO}^{+}\right), 77\left(100 \%, \mathrm{Ph}^{+}\right)$. Isotopic pattern, $\mathrm{m} / \mathrm{e}=394\left(3 \%, \mathrm{M}^{+}\right), 357\left(2 \%, \mathrm{M}^{+}-\mathrm{Cl}\right), 317$ $\left(6 \%, \mathrm{M}^{+}-\mathrm{Ph}\right)$.

Anal. Calcd. for $\mathrm{C}_{20} \mathrm{H}_{11} \mathrm{O}_{2} \mathrm{Cl}_{3}$ : C, 61.65; H, 2.85; O, 8.22 Found: $\mathrm{C}, 61.55 ; \mathrm{H}, 2.77$; O, 8.15

3',3'-Diphenyl-4,5,6,7-tetrachlorophthalide (12): White crystals m.p.: $175-77^{0} \mathrm{C}$, yield 0.33g (10\%), $\mathrm{R}_{\mathrm{f}} 0.50$ (silicagel, benzene : pet. ether 2:3). IR: 3000 (s, Ar-H stretching), $1760(\mathrm{~s},>\mathrm{C}=\mathrm{O}), 1600$ and $1570(\mathrm{w},>\mathrm{C}=\mathrm{C}<$, aromatic ring), 790 and $780(\mathrm{w}, \mathrm{Ar}-\mathrm{H}$, bending) $\mathrm{cm}^{-1}$; UV: $\lambda_{\max }(\log \varepsilon)=252(4.33), 162(3.25) \mathrm{nm} ;{ }^{1} \mathrm{H} \mathrm{NMR}\left(\mathrm{CDCl}_{3}, \delta, 500\right.$ MHz); 7.48 (4H, d, J=7.4, H-2",6"), 7.09 (4H, t, J=7.1, H-3",5"), 7.23 (2H, d, J=7.3, H4 "), ppm; ${ }^{13} \mathrm{C}$ NMR $\left(\mathrm{CDCl}_{3}, \delta, 125.5 \mathrm{MHz}\right): 190.45$ (phthalide), 140.15 (C-1), 139.45 (C2), 137.64 (C-4), 137.15 (C-5), 135.56 (C-6), 128.85 (C-7), 80.35 (C-3), 139.35 (C-1"), $128.57\left(\mathrm{C}-2^{\prime \prime}, 6^{\prime \prime}\right), 128.28\left(\mathrm{C}-3^{\prime \prime}, 5^{\prime \prime}\right), 127.95\left(\mathrm{C}-4^{\prime \prime}\right) \mathrm{ppm}$; MS (EI): m/e = $422\left(5 \%, \mathrm{M}^{+}\right)$, $394\left(10 \%, \mathrm{M}^{+}-\mathrm{CO}\right), 360\left(5 \%, \mathrm{M}^{+}-\mathrm{CO}-\mathrm{Cl}\right), 345(28 \%, \mathrm{M}-\mathrm{Ph})^{+}, 268(5 \%, \mathrm{M}-2 \mathrm{Ph})^{+}, 256$ $(10 \%, \mathrm{M}-2 \mathrm{Ph}-\mathrm{Cl})^{+}, 105(100 \%, \mathrm{PhCO})^{+}, 77(82 \%, \mathrm{Ph})^{+}$. Isotopic pattern, $\mathrm{m} / \mathrm{e}=430(2 \%$, $\left.\mathrm{M}^{+}\right), 402\left(3 \%, \mathrm{M}^{+}-\mathrm{Cl}\right), 366\left(2 \%, \mathrm{M}^{+}-\mathrm{CO}-\mathrm{Cl}\right)$.

Anal. Calcd. for $\mathrm{C}_{20} \mathrm{H}_{10} \mathrm{O}_{2} \mathrm{Cl}_{4}$ : C, 56.64; H, 2.38; O, 7.55 Found: C, 56.57; H, 2.31; O, 7.48

\section{Acknowledgement}

The author thanks the Bangladesh University of Engineering and Technology Dhaka for financial assistance. The author also thanks the Department of Organic Chemistry, Chalmers University of Technology, Goteborg, Sweden and Department of Chemistry, University of Dhaka for taking some spectra.

\section{References}

1. M. T. Rahman and S. K. Nahar. Organomet. Chem, 1992, 425, 201.

2. V. A. Freund, Ann, 1861, 18, 3 .

3. P. Andre, Compt. Rend, 1910, 151, 76. 
4. G. V. Bechi, Ber, 1879, 12, 463.

5. H. Gilman and J. Starley, Rec. trav. Chem, 1936, 55, 821.

6. H. Gilman, E. Kirby, Rec. Trav. Chem, 1929, 48, 155.

7. S. H. Bertz, S. Cope, M. Murphy, C. A. Ogle, B. J. Taylor, J. Amer. Chem. Soc, 2007, 129, 7208.

8. H. Hu, J. P. Snyder, J. Amer. Chem. Soc, 2007, 129, 7210.

9. J. H. Kim, S. H. Jung, Tetrahedron Letters, 2007, 48, 4243.

10. Caprio, Vittorio, Letters in Organic Chemistry, 2006, 3, 339-349.

11. G. Helmchen, M. Ernst, G. Paradies, Pure Appl. Chem, 2004, 76, 495-506.

12. M. T. Rahman and S. K. Nahar, Organomet. Chem, 1967, 129, 133.

13. J. H. P. Tyman and A. A. Najam, Spectrochimica Acta, 1977, 33A, 479.

14. M. T. Rahman and H. Gilman, J. Indian Chem. Soc, 1974, 51, 1018.

15. H. Gilman and F. Schulze, J. Amer. Chem. Soc, 1925, 47, 2002.

16. M. T. Rahman, A. K. M. Hoque, I. Siddique, D. A. N Chowdhury, S. K. Nahar and S. L, Sahin, J. Organomet. Chem, 1980, 188, 297.

(Received : 05 November, 2007; Accepted: 29 November, 2012) 